\title{
ESTUDO PARA DETERMINAR A RESISTÊNCIA MECÂNICA A FLEXÃO DE MATERIAIS CERÂMICOS, COM USO DISTRIBUIÇÃO DE WEIBULL*
}

\author{
Enir da Silva Fonseca ${ }^{1}$ \\ Fernando Gonzales Tavares ${ }^{2}$ \\ Thyrson Niênio Rodrigues Sousa ${ }^{3}$ \\ Claudio Pereira Diogo ${ }^{4}$ \\ Willy Ank de Morais ${ }^{5}$
}

\section{Resumo}

Este trabalho tem como objetivo principal analisar os dados obtidos através dos ensaios a flexão em 40 corpos de provas cerâmicos preparados de acordo com a NBR 13818:1997 e aplicar uma probabilidade estatística para cálculo da resistência à fratura. O modulo de resistência à flexão para a ruptura de cada corpo de prova foi calculado de acordo com a fórmula apresentada no anexo $\mathrm{C}$ da norma citada, e aplicada posteriormente no lote à estatística para distribuição de Weibull. Os resultados obtidos não apresentaram uma variação significativa na resistência mecânica à flexão para os corpos de provas sem umidade em relação aos submetidos à imersão em água.

Palavras-chave: Placa cerâmica; Resistência mecânica; Weibull.

\section{STUDY TO DETERMINE THE MECHANICAL STRENGTH THE BENDING OF CERAMIC MATERIALS WITH DISTRIBUTION OF USE WEIBULL}

\section{Abstract}

This work aims to analyze the data obtained from the tests flexion in 40 bodies of ceramic evidence prepared according to NBR 13818: 1997 and apply a statistical probability to calculate the fracture toughness. The flexural modulus of rupture for each specimen was calculated according to the formula given in Annex $\mathrm{C}$ of this standard, and applied later in the batch statistic for Weibull distribution. The results showed no significant variation in flexural strength for the test samples without moisture in those submitted to immersion in water.

Keywords: Ceramic plate; Mechanical strength; Weibull.

1 Mestrando em Engenharia Mecânica na UNISANTA, Pós-Graduado em Engenharia da Computação pela UFU e Bacharel em Ciências da Computação pela UNICEB. Santos, SP, Brasil.

2 Mestrando em Engenharia Mecânica pela UNISANTA, Pós-Graduação para o Ensino Superior pela UNIMES, aperfeiçoamento em Estatística no IME-USP e Bacharel em matemática. Santos, SP, Brasil.

3 Mestrando em Engenharia Mecânica na UNISANTA, Pós-Graduado em Engenharia da Segurança do Trabalho pela UNISANTA e Graduado em Engenharia Industrial Mecânica pela UNISANTA. Santos, SP, Brasil.

4 Mestre em Engenharia Metalúrgica e de Materiais pela PUC-Rio, graduação em Engenharia Metalúrgica pela EM-UFOP 1995, técnico em Metalurgia pela ETFOP. Professor Adjunto da Faculdade de Engenharia da UNISANTA. Santos, SP, Brasil.

5 Bacharelando em Engenharia de Produção pela UNIMONTE. Santos, SP, Brasil. 


\section{INTRODUÇÃO}

A cerâmica é o material artificial mais antigo produzido pelo homem. Do grego "kéramos" ("terra queimada" ou "argila queimada"), é um material de grande resistência, frequentemente encontrado em escavações arqueológicas. Pesquisas apontam que a cerâmica é produzida há cerca de 10-15 mil anos. [1] De acordo com Associação Brasileira de Cerâmica o material cerâmico é definido como "todos os materiais inorgânicos, não metálicos, obtidos geralmente após tratamento térmico em temperaturas elevadas", e que devida a sua amplitude de matérias-primas e possibilidades de uso, são classificadas em: Cerâmica Vermelha, Materiais de Revestimento (Placas Cerâmicas), Cerâmica Branca, Materiais Refratários, Isolantes Térmicos, Abrasivos, Vidro, Cimento e Cal. [2]

A qualidade do revestimento nacional e a agilidade dos fabricantes em acompanhar as tendências internacionais têm assegurado o crescimento das vendas. O Brasil é hoje o segundo produtor e consumidor mundial de Revestimento Cerâmico, ficando atrás apenas de China, e é o quarto exportador mundial. As estimativas para a produção de 2014 é de 917,2 milhões de metros cúbicos superior em 5,29\% em relação a 2013 que produziu 871,1 milhões de metros cúbicos. [1] Em 2013, foram produzidos 871,6 milhões de metros quadrados, para uma capacidade instalada de 1.023.37 milhões de metros quadrados. As vendas totais atingiram 900,84 milhões de metros quadrados, sendo 837,52 milhões de metros quadrados vendidos no mercado interno e 63,32 milhões de metros quadrados exportados [1]. Defeitos na microestrutura de materiais frágeis, normalmente não apresentam valores precisos de resistência quando submetidos a ensaios de resistência mecânicos, os dados obtidos sofrem significativa dispersão nos resultados obtidos, quando comparados com materiais dúcteis. Os materiais cerâmicos de uma maneira geral devido à sua microestrutura, quando apresentam um pequeno defeito em um ponto podem comprometer toda a sua estrutura, eles são como correntes: um elo fraco e ela rompem.

Objetivando predizer o comportamento de materiais frágeis em geral, em 1983, o físico sueco Wallodi Weibull desenvolveu um método estatístico que vem sendo empregado com sucesso não apenas neste tipo de estudo, mas também em outras áreas, como, por exemplo, Biologia, Controle de Processos, Eletricidade (WANG et al, 1997) [9], entre outros. Neste trabalho foi utilizada a distribuição de Weibull para descrever o comportamento desta propriedade, dado que é bastante utilizada em análise de falhas, resistência à fratura e comportamento em fadiga. Através de dados obtidos em ensaios de flexão, foi demonstrado o método de Weibull com dois e três parâmetros. Um dos parâmetros de classificação dos pisos cerâmicos é a absorção de água, que tem influência direta sobre outras propriedades como, por exemplo, a resistência mecânica á flexão, a qual o seu valor aumenta, à medida que a absorção diminui. Este artigo, que tem como objetivo principal Determinar a Resistência Mecânica a Flexão de Materiais Cerâmicos, Comparando a Confiabilidade do lote com a Estatística de Weibull.

\section{MATERIAIS E MÉTODOS}

Foram selecionados 40 corpos de provas com o comprimento de $29,8 \mathrm{~mm}$, largura de $14,8 \mathrm{~mm}$ e espessura de $7,8 \mathrm{~mm}$, apresentados na Figura 1 e escolhidos aleatoriamente. 


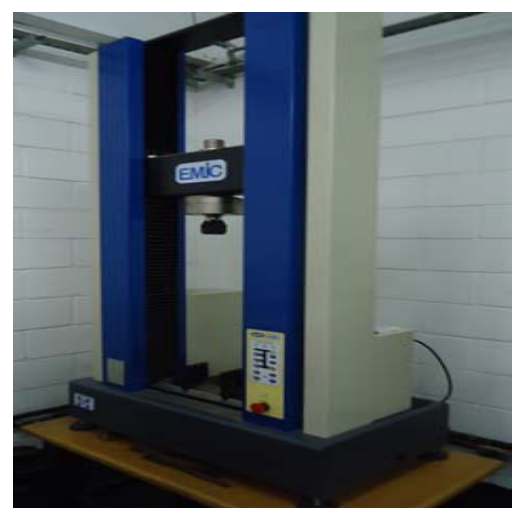

Figura 1. À esquerda: amostras empregadas no estudo. À direita: máquina de Ensaios -EMIC DL5000.

A realização dos ensaios a flexão e resistência dos pisos cerâmicos a três pontos foram estabelecidas de acordo com a norma NBR 13818:1997, e os corpos de prova ensaiados foram submetidos à compreensão na máquina Universal de Ensaios, eletromecânica e microprocessada EMIC modelo DL5000, como apresentado na Figura 1, com o Programa Tesc versão 3.01.

Os pisos cerâmicos conduzidos a uma estufa a $110^{\circ} \mathrm{C}$ por 26 horas, mostrada na Figura 2, e depois de concluído esse tempo foi resfriada a temperatura ambiente dentro do dessecador e submetida às provas em um intervalo de uma hora na máquina de ensaios.
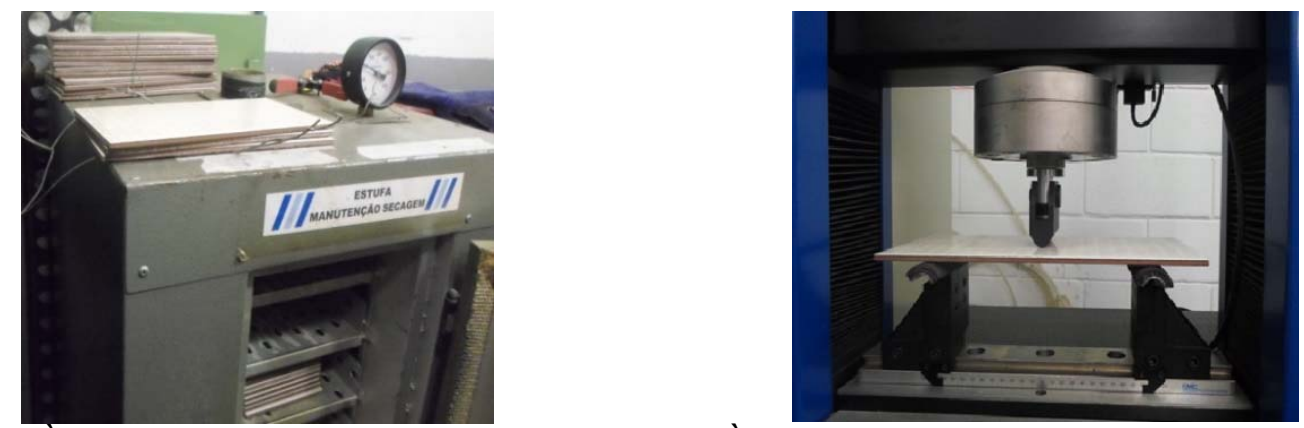

Figura 2. A esquerda: Estufa para secagem dos CPs. À direita: amostra pronta para o ensaio.

As amostras foram medidas com um paquímetro analógico Mitutoyo de resolução $0,05 \mathrm{~mm}$ e posicionadas na máquina universal de ensaios de acordo com os padrões apresentados na tabela C.1 "Dimensões dos apoios e da barra", descritos no anexo C da NBR 13818 e ensaiadas conforme ilustrado na Figura 2.

Para alcançar os resultados os ensaios foram realizados nos laboratórios de Engenharia Mecânica da UNISANTA (Universidade Santa Cecília) e o tratamento térmico realizado em uma estufa da INSPEBRAS ${ }^{\circledR}$ [5], de acordo com a NBR 13818:1997 "Placas cerâmicas para revestimento - Especificação e métodos de ensaios" apresenta em seu anexo C [6].

Ao analisar a influência da absorção da umidade na resistência a flexão de piso cerâmico, seguiu-se o estabelecido na norma NBR 13818 e os corpos de prova a serem ensaiados foram conduzidos a uma estufa a $110^{\circ} \mathrm{C}$ por 26 horas. Concluído esse tempo, os pisos cerâmicos foram divididos em 3 grupos com 3 corpos de prova para ensaio iniciais e ajustes da velocidade da máquina de ensaios, 34 corpos de provas para ensaios de acordo com a norma citada e mais 3 corpos de prova submetidos a imersão em água por 30 minutos. Os ensaios de flexão realizados em três pontos e calculado sua resistência à flexão seguindo a Equação (1): 


$$
M R F=\sigma=\frac{3 \cdot F \cdot L}{2 \cdot b \cdot e^{2}}
$$

Onde: MRF ou $\sigma \quad$ - Modulo de resistência à flexão

$$
\begin{array}{ll}
\text { F } & \text { - Força de ruptura em Newtons } \\
\text { L } & \text { - Distância da barra de apoio em milímetros } \\
\text { b } & \text { Largura do corpo de prova ao longo da ruptura em milímetros } \\
\text { e } & \text { - Espessura do corpo de prova em milímetros }
\end{array}
$$

Para os ensaios os CP's utilizados foram submetidos de forma aleatória e identificados no início e durante o processo, sendo o primeiro procedimento uma inspeção visual para detectar possíveis avarias ou fraturas que inviabilizassem o experimento. Os cálculos e gráficos da função Densidade da distribuição Weibull foram construídos com o uso do software Minitab 17.

\section{RESULTADOS E DISCUSSÃO}

Ao calcular a tensão (1) do grupo inicial de 3 corpos de provas em velocidade a taxa de $0,04 \mathrm{MPa} / \mathrm{s}$ obteve-se o gráfico mostrado na Figura 3 que apresenta a relação entre as medidas medias de Flexão e Tensão dos CP's 01 ao 03 e, por conseguinte tal gráfico representa os valores médios de resistências dos três corpos de provas com seus respectivos momentos de ruptura. A variabilidade do conjunto de dados e CV são inferiores a $32 \%$.

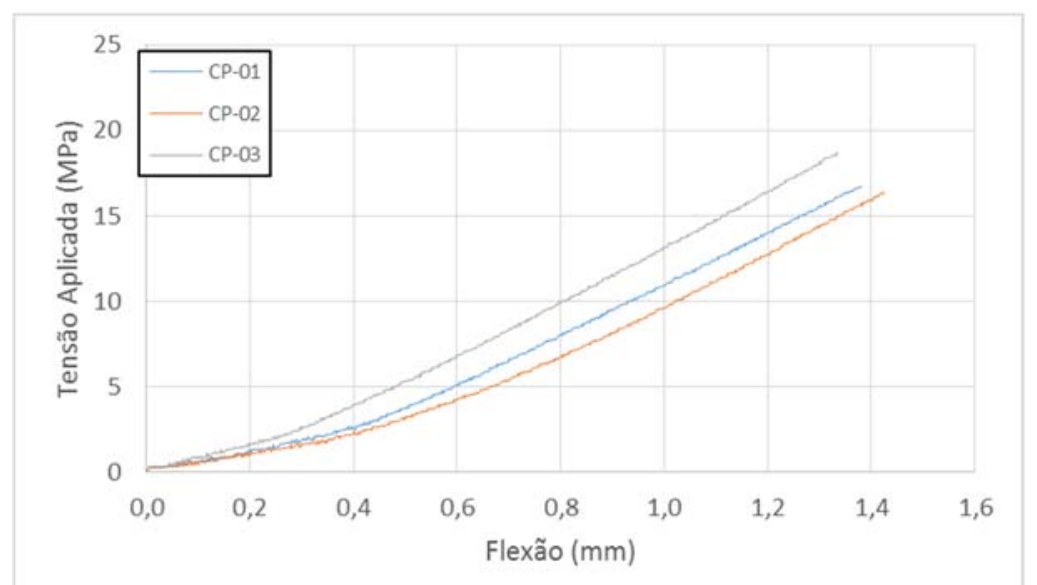

Figura 3. Corpos de prova (01-03) ensaiados com velocidade 0,04MPa/s.

Os ensaios para os CP's 04 até o 37 foi empregado uma velocidade a taxa de $0,21 \mathrm{MPa} / \mathrm{s}$ que se obteve as medidas médias de flexão e tensão apresentada no gráfico da Figura 4. Foi verificada a ausência de corpos de prova com comportamento muito discrepante em relação aos demais. Observa-se que embora alterada a velocidade que permite o movimento das células de carga, os resultados obtidos das amostras sofrem alteração demonstrando uma variação na composição dos CP's. 


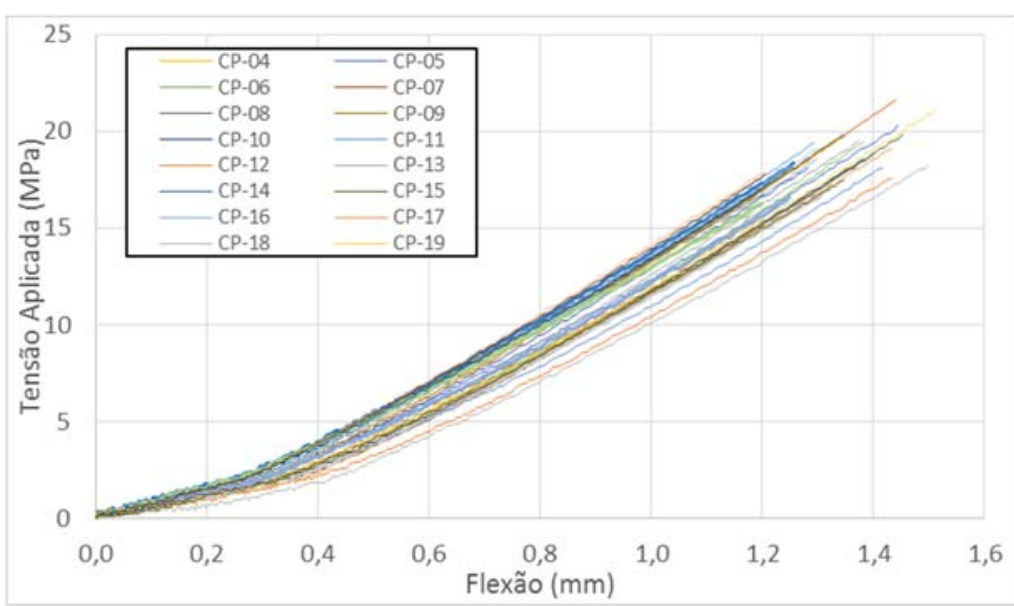

Figura 4. Corpos de prova (04-37) ensaiados com velocidade 0,21MPa/s.

Observa-se que embora alterada a velocidade que permite o movimento das células de carga, os resultados obtidos das amostras sofrem alteração demonstrando uma variação na composição dos CP's. Para os ensaios para dos CP's 38 ao 40 que foram submetidos a imersão em água por 30 minutos, foi empregado uma velocidade a taxa de $0,21 \mathrm{MPa} / \mathrm{s}$ e o resultado é apresentado no gráfico apresentado na Figura 5 exibindo a relação entre as medidas medias de flexão e tensão dos CP's 38 ao 40.

Figura 5. Corpos de prova (38-43) ensaiados com velocidade $0,21 \mathrm{MPa} / \mathrm{s}$ (após a imersão em água).

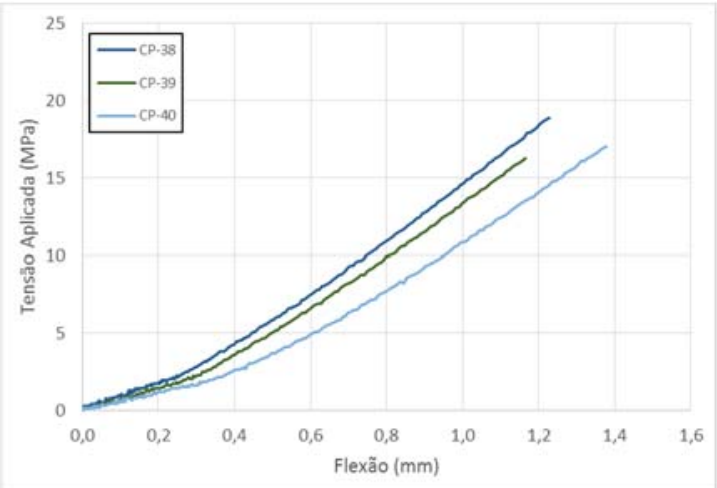

\subsection{Análise dos Dados}

A análise de dados realizada neste é o método de Weibull que é o mesmo de IMASAVA [8] descrito a seguir e que consiste basicamente, de cinco passos:

1 - Realizar "n" ensaios de flexão em 3 pontos;

2 - Preparar os dados (cálculo das tensões);

3 - Aplicar o método de Weibull;

4 - Traçar o gráfico, com os valores de tensão e as curvas obtidas pela regressão;

5 - Analisar os resultados.

\subsection{Realização dos Ensaios}

A parte experimental na análise da resistência mecânica dos corpos de provas cerâmicos através dos ensaios de flexão e que pode influenciar significativamente os resultados é o número de amostras a serem testadas. 
Segundo o método de Weibull, não é necessário um número de amostras muito grande para se atingir resultados confiáveis. Neste sentido, o número mínimo de ensaios a serem efetuados, de acordo com IMASAVA [8], para que se obtenha um resultado confiável, são 10 ensaios, sendo que o ideal, ainda de acordo com os mesmos autores está situado entre 30 a 40 . Alguns cuidados que foram tomados antes e durante os ensaios:

- Os ensaios foram realizados de uma só vez, da mesma forma e pelas mesmas pessoas a fim de reduzir a tendência de dispersão dos resultados.

- Os corpos de prova foram mantidos em um forno na temperatura de $110^{\circ} \mathrm{C}$ e após serem retirados, permaneceram dentro do dessecado para retornarem a temperatura ambiente e ensaiados;

- A máquina de ensaio foi calibrada de modo que a velocidade do ensaio seja pequena, de maneira que os resultados não sejam mascarados devido ao impacto;

- Todo o processo realizado com boa estabilidade operacional.

Com o objetivo de avaliar a relevância e os cuidados a serem tomados no controle do nível de umidade dos corpos de prova e velocidade utilizada na realização dos ensaios de flexão em três pontos, foram realizados três tipos de experimentos em amostras de piso cerâmico descriminados a seguir:

$1^{\circ}$ - Utilizado 3 corpos de prova com velocidade $0,04 \mathrm{MPa} / \mathrm{s}$ após atingir a temperatura ambiente;

$2^{\circ}$ - Utilizado 34 corpos de prova com velocidade $0,21 \mathrm{MPa} / \mathrm{s}$ após atingir a temperatura ambiente;

$3^{\circ}$ - Utilizado 3 corpos de prova com velocidade $0,21 \mathrm{MPa} / \mathrm{s}$ após serem imersos em água durante 1 hora.

Nas Tabelas 1, 2 e 3 são apresentados os dados de cada grupo e os resultados obtidos nos ensaios. E a partir destes dados foi calculada a tensão de ruptura em cada ensaio, segundo a Equação (1), para ensaio de flexão em três pontos os quais também são mostrados nas Tabelas 1,2 e 3.

Tabela 1. Dados e resultados do $1^{\circ}$ ensaio Velocidade de 0,04MPa/s.

\begin{tabular}{ccccc}
\hline $\mathbf{C P}$ & $\mathbf{t}(\mathbf{s})$ & $\mathbf{\delta}(\mathbf{m m})$ & $\mathbf{F ( N )}$ & $\boldsymbol{\sigma}(\mathbf{M P a})$ \\
\hline 1 & 414,0 & 1,37992 & 501,41 & 16,70560 \\
2 & 427,5 & 1,42497 & 491,65 & 16,38047 \\
3 & 400,5 & 1,33499 & 560,02 & 18,65838 \\
\hline
\end{tabular}

Tabela 3. Dados e resultados do $3^{\circ}$ ensaio Velocidade de $0,21 \mathrm{MPa} / \mathrm{s}$.

\begin{tabular}{ccccc}
\hline $\mathbf{C P}$ & $\mathbf{t}(\mathbf{s})$ & $\boldsymbol{\delta}(\mathbf{m m})$ & $\mathbf{F ( N )}$ & $\boldsymbol{\sigma}(\mathbf{M P a})$ \\
\hline 38 & 73,7 & 1,22801 & 566,53 & 18,8753 \\
39 & 69,9 & 1,16495 & 488,39 & 16,2719 \\
40 & 82,7 & 1,37853 & 511,18 & 17,0312 \\
\hline
\end{tabular}

Tabela 2. Dados e resultados do $2^{\circ}$ ensaio Velocidade de 0,21MPa/s.

\begin{tabular}{ccccc}
\hline $\mathbf{C P}$ & $\mathbf{t}(\mathbf{s})$ & $\boldsymbol{\delta}(\mathbf{m m})$ & $\mathbf{F}(\mathbf{N})$ & $\boldsymbol{\sigma}(\mathbf{M P a})$ \\
\hline 4 & 77,8 & 1,29641 & 498,16 & 16,5974 \\
5 & 87,2 & 1,45337 & 612,12 & 20,3942 \\
6 & 80,8 & 1,34720 & 592,58 & 19,7432 \\
$\ldots$ & $\ldots$ & $\ldots$ & $\ldots$ & $\ldots$ \\
35 & 72,3 & 1,20453 & 533,97 & 17,7905 \\
36 & 83,0 & 1,38278 & 556,77 & 18,5501 \\
37 & 80,8 & 1,34722 & 524,21 & 17,4653 \\
\hline
\end{tabular}




\subsection{Análise Exploratória dos Dados}

De acordo com NIST [7], a análise exploratória de dados (AED) emprega uma variedade de técnicas gráficas para: maximizar o conhecimento sobre um conjunto de dados; investigar hipóteses sobre a estrutura dos dados; detectar outliers; examinar relações entre as variáveis. Para explorar adequadamente a natureza dos dados e suas propriedades, é importante que seja feita a AED, ilustrada na Tabela 4, antes do uso de certas ferramentas estatísticas, para evitar erros grosseiros, e a análise parcial com a geração de resultados duvidosos.

Tabela 4. Estatísticas Descritivas: $\sigma(\mathrm{MPa}) ; \delta(\mathrm{mm})$.

\begin{tabular}{lcccccccccc}
\hline Variável & \multirow{N}{*}{} & Média & $\begin{array}{c}\text { EP } \\
\text { Medio }\end{array}$ & DesvPad & CoefVar & Mín & Q1 & Mediana & Q3 & Máx \\
\hline$\sigma(\mathrm{MPa})$ & 34 & 18,381 & 0,238 & 1,387 & 7,55 & 16,055 & 17,194 & 18,333 & 19,445 & 21,587 \\
$\delta(\mathrm{mm})$ & 34 & 1,331 & 0,016 & 0,092 & 6,92 & 1,197 & 1,254 & 1,331 & 1,403 & 1,513 \\
\hline
\end{tabular}

Fez-se a análise de dados visando revelar outliers através de técnica gráfica como Box-Plot (caixa de bigodes), mostrados nos gráficos da Figura 6, e teste de Razão de Dixon apresentados na Tabela 5. O Box-Plot é uma excelente ferramenta, pois permiti visualizar a locação, a dispersão, a simetria, os limites de outliers e os próprios outliers, independentemente da forma da distribuição de um conjunto de dados. O Box-Plot é construído com base na mediana e nos quartis associados ao conjunto de dados, o que o torna resistente a valores perturbadores dentro da faixa de limites de outliers e, consequentemente, atraente em análise exploratória de dados [4].

Tabela 5. Teste de Razão (r22) de Dixon.

\begin{tabular}{lccccccccc}
\hline Variável & $\mathrm{N}$ & Mín & $\mathrm{x}[2]$ & $\mathrm{x}[5]$ & $\mathrm{x}[\mathrm{N}-2]$ & $\mathrm{x}[\mathrm{N}-1]$ & Máx & $\mathrm{r} 22$ & $\mathrm{p}$ \\
\hline$\sigma(\mathrm{MPa})$ & 34 & 16,055 & 16,272 & 16,597 & 20,394 & 21,154 & 21,587 & 0,24 & 0,523 \\
$\delta(\mathrm{mm})$ & 34 & 1,1972 & 1,198 & 1,1989 & 1,4536 & 1,498 & 1,513 & 0,19 & 0,847 \\
\hline
\end{tabular}
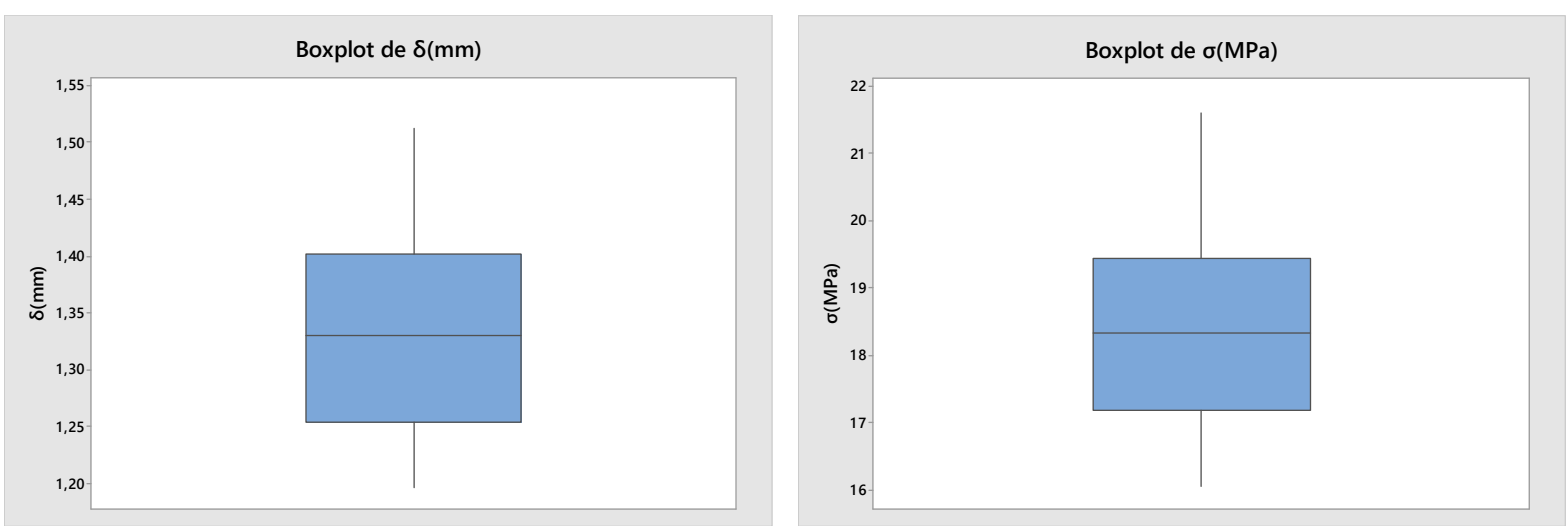

Figura 6. À esquerda: dados do conjunto Flexão. À direita: dados do conjunto Tensão.

Os valores r22 no Teste de Razão de Dixon, são obtidos considerando-se como hipótese nula $(\mathrm{H} 0)$ : Todos os valores da amostra são da mesma população normalmente distribuída. Sua hipótese alternativa $(\mathrm{H} 1)$ é: Um dos valores da amostra não é da mesma população normalmente distribuída. Dixon [10] observou que as maiores amostras de uma população normal têm maior probabilidade de incluir valores extremos, por essa razão analisamos a presença de outliers no 
conjunto de dados utilizando a razão $\mathrm{Q}$ de Dixon $\mathrm{r}_{22}$ para amostras com tamanho $\mathrm{N}$ $>=14$ dados. Não foi detectado nenhum outlier no nível de significância 5\%.

Barnett\&Lewis [3] definiram outlier como sendo uma observação que parece ser inconsistente com os demais elementos do conjunto de dados. Os outliers podem indicar características importantes sobre um modelo, como modelo incompatível com os dados ou omissão de variáveis importantes. Os gráficos da Figura 6 apresentam um traço superior mais longo o que indica uma leve assimetria positiva, a lateral direita da distribuição é mais longa do que a lateral esquerda. Nenhum outlier foi identificado em nenhum dos dois conjuntos de dados

\subsection{Descrição do Método de Weibull.}

Ordenando-se as tensões de ruptura calculadas no $2^{\circ}$ ensaio, atribuímos um valor de probabilidade para romper o corpo de prova, e de acordo com a Equação (2), método de Kaplan e Meier utilizado pelo software Minitab. Verifica-se que o menor valor de tensão terá a menor probabilidade, e o maior valor de tensão terá a maior probabilidade.

$$
P_{i}=\frac{i}{n}
$$

Desta maneira, está-se sugerindo que um valor de tensão baixo, naquelas condições do ensaio, possui uma probabilidade baixa de romper o corpo de prova, e que um valor alto possui uma probabilidade maior de romper o material. Os resultados desta etapa são mostrados na Tabela 6.

Tabela 6. Probabilidades dos CP s do $2^{\circ}$ ensaio.

\begin{tabular}{cccccccc}
\hline CP & $\boldsymbol{\sigma}(\mathbf{M P a})$ & Od. & Prob. & CP & $\boldsymbol{\sigma}(\mathbf{M P a})$ & Od. & Prob. \\
\hline 19 & 16,05496 & 1 & 0,03 & 8 & 18,33287 & 18 & 0,53 \\
33 & 16,27186 & 2 & 0,06 & 22 & 18,33287 & 19 & 0,56 \\
4 & 16,59737 & 3 & 0,09 & 16 & 18,55010 & 20 & 0,59 \\
$\ldots$ & $\ldots$ & $\ldots$ & $\ldots$ & $\ldots$ & $\ldots$ & $\ldots$. & $\ldots$ \\
26 & 18,11597 & 15 & 0,44 & 5 & 20,39421 & 32 & 0,94 \\
18 & 18,22459 & 16 & 0,47 & 31 & 21,15351 & 33 & 0,97 \\
7 & 18,33287 & 17 & 0,50 & 23 & 21,58730 & 34 & 0,99 \\
\hline
\end{tabular}

Weibull propôs esta distribuição para descrever o tempo de vida de materiais sob cargas que causavam fadiga e fraturas nestes materiais. Considerando-se a distribuição das falhas na forma da Equação (3), para $x>=x_{u}, x_{0}>0$ e $m>0$, onde $\boldsymbol{P}$ é a probabilidade de falha ao se considerar o fator $\mathrm{x}$, resistência do piso cerâmico.

$$
P(x)=1-\exp \left[-\left(\frac{x-x_{u}}{x_{0}}\right)^{m}\right]
$$

Onde: $x_{0}$ é o fator de escala, que é o valor característico da distribuição, tal como o tempo de vida, a resistência mecânica ou a carga; $\mathbf{m}$ é o parâmetro de forma da distribuição, mais conhecido como coeficiente de Weibull. Este valor controla a 
variância dos valores medidos; quanto maior seu valor, mais estreita é a distribuição dos valores medidos, e mais alto é o pico; $\mathbf{x}_{u}$ é chamado parâmetro de localização, que é o menor valor característico do parâmetro medido. A probabilidade de ocorrer uma falha, se, por exemplo, a tensão aplicada for menor que $\mathbf{x u}_{u}$ é zero. Desta forma, a resistência medida será sempre o menor valor de um conjunto de valores possíveis. Se os ensaios forem repetidos, outros valores mínimos serão obtidos, assim a resistência medida pode ser considerada um valor aleatório.

A Equação (3) é chamada "Distribuição de Weibull com 3 Parâmetros". Assumindo que o menor valor possível de resistência de qualquer corpo cerâmico é zero, $\mathbf{x}_{\mathbf{u}}=0$, a distribuição resultante é chamada de "Distribuição de Weibull com 2 parâmetros, Equação (4).

$$
P(x)=1-\exp \left[-\left(\frac{x}{x_{0}}\right)^{m}\right]
$$

\subsection{Cálculo dos Valores da Distribuição de Weibull com dois Parâmetros}

Esta forma da distribuição de Weibull é frequentemente usada quando o menor valor da variável aleatória pode ser assumido como sendo zero. Os parâmetros da distribuição de Weibull para um determinado conjunto de dados pode ser estimado através de vários métodos diferentes. Neste artigo usamos método de regressão linear. Através do rearranjo da equação (4) e da aplicação de logaritmo natural duas vezes, obtém-se a Equação (5), de caráter linear:

$$
\begin{aligned}
& \mathrm{P}(\mathrm{x})=1-\mathrm{e}^{-\left(\frac{\mathrm{x}}{\mathrm{x}_{0}}\right)^{\mathrm{m}}} \rightarrow \mathrm{P}(\mathrm{x})-1=-\mathrm{e}^{-\left(\frac{\mathrm{x}}{\mathrm{x}_{0}}\right)^{\mathrm{m}}} \rightarrow \\
& 1-\mathrm{P}(\mathrm{x})=\mathrm{e}^{-\left(\frac{\mathrm{x}}{\mathrm{x}_{0}}\right)^{\mathrm{m}}} \rightarrow \frac{1}{1-\mathrm{P}(\mathrm{x})}=\mathrm{e}^{\left(\frac{\mathrm{x}}{\mathrm{x}_{0}}\right)^{\mathrm{m}}} \rightarrow \\
& \ln \left[\ln \left(\frac{1}{1-\mathrm{P}(\mathrm{x})}\right)\right]=\ln \left[\ln \mathrm{e}^{\left(\frac{\mathrm{x}}{\mathrm{x}_{0}}\right)^{\mathrm{m}}}\right] \rightarrow \ln \left[\ln \left(\frac{1}{1-\mathrm{P}(\mathrm{x})}\right)\right]=\ln \left(\frac{\mathrm{x}}{\mathrm{x}_{0}}\right)^{\mathrm{m}} \rightarrow \\
& \ln \left[\ln \left(\frac{1}{1-\mathrm{P}(\mathrm{x})}\right)\right]=\mathrm{m}\left[\ln (\mathrm{x})-\ln \left(\mathrm{x}_{0}\right)\right] \rightarrow \\
& \ln \left[\ln \left(\frac{1}{1-\mathrm{P}(\mathrm{x})}\right)\right]=\mathrm{m} \ln (\mathrm{x})-\mathrm{m} \ln \left(\mathrm{x}_{0}\right)
\end{aligned}
$$

Aplicando-se a Equação (5) ao estudo da resistência mecânica de materiais frágeis, obtém-se a Equação (6). A partir desta, considerando não haver variação de volume, obtem-se a Equação Final (7).

$$
\ln \left[\ln \left(\frac{1}{1-P\left(\sigma_{P}\right)}\right)\right]=m \ln \sigma_{P}-m \ln \sigma_{0}+\ln \left(\frac{V}{V_{0}}\right)
$$




$$
\ln \left[\ln \left(\frac{1}{1-P\left(\sigma_{P}\right)}\right)\right]=m \ln \sigma_{P}-m \ln \sigma_{0}
$$

Onde: $\sigma_{\mathbf{P}}$ é a tensão de ruptura do material cerâmico associada a probabilidade $\mathbf{P}$ e $\sigma_{0}$ é a resistência média do material.

Para a determinação dos coeficientes da equação (7), é necessário ainda calcular os valores de $\ln \left(\sigma_{i}\right)$ e $\ln \left[\ln \left(1 /\left(1-P_{i}\right)\right]\right.$ apresentados na Tabela 7.

Tabela 7. Dados calculados para Regressão Linear do $2^{\circ}$ ensaio.

\begin{tabular}{cccccc}
\hline $\mathbf{C P}$ & $\ln \boldsymbol{\sigma}$ & $\operatorname{In}[\operatorname{In}(\mathbf{1} /(\mathbf{1 - P i})]$ & $\mathbf{C P}$ & $\operatorname{In} \boldsymbol{\sigma}$ & $\operatorname{In}[\ln (\mathbf{1} /(\mathbf{1}-\mathbf{P i})]$ \\
\hline 19 & 2,77602 & $-3,51147$ & 8 & 2,90870 & $-0,28267$ \\
33 & 2,78944 & $-2,80305$ & 22 & 2,90870 & $-0,20051$ \\
4 & 2,80924 & $-2,38192$ & 16 & 2,92048 & $-0,11957$ \\
$\ldots$ & $\ldots$ & $\ldots$ & $\ldots$ & $\ldots$ & $\ldots$ \\
26 & 2,89679 & $-0,54142$ & 5 & 3,01525 & 1,04141 \\
18 & 2,90277 & $-0,45257$ & 31 & 3,05181 & 1,26027 \\
7 & 2,90870 & $-0,36651$ & 23 & 3,07211 & 1,76284 \\
\hline
\end{tabular}

Para obter a reta ajustada, de onde se determina o módulo de Weibull $\mathbf{m}$ (coeficiente angular A) e o fator de escala $\mathbf{x}_{\mathbf{0}}$ calculado a partir do coeficiente linear $\mathbf{B}$ como:

$$
x_{0}=e^{-(B / m)}
$$

Considerando a equação geral da reta de regressão como:

$$
Y=A X+B
$$

$$
\begin{aligned}
& \text { Onde: } \ln \left[\ln \left(\frac{1}{1-P\left(\sigma_{P}\right)}\right)\right]=\text { variável dependentede }(Y) \\
& \ln \sigma_{P}=\text { variável independentede }(X) \\
& m=\text { responsável pelainclinaçãoda reta }(A) \\
& -m \ln \sigma_{0}=\text { coeficiente linear (B) }
\end{aligned}
$$

Com a equação da reta ajustada, mostrada na Figura 7 , podem ser determinados os valores dos dois parâmetros de Weibull, módulo $m=13,71$ e o fator de escala $x_{0}=$ 19,03 usados na construção dos gráficos de distribuição de probabilidades da distribuição acumulada de Weibull mostrado na Figura 7 e o gráfico de distribuição de probabilidades, mostrado na Figura 8.

\subsection{Cálculo dos Valores da Distribuição de Weibull com Três Parâmetros}

Utilizando a equação (3) do modelo de distribuição de Weibull com três parâmetros e fazendo as devidas alterações para utilizar o método de regressão linear e através do rearranjo da Equação (4) e da aplicação de logaritmo natural duas vezes, obtémse a equação linear (10): 


$$
\ln \left[\ln \left(\frac{1}{1-P(\sigma)}\right)\right]=m \ln \left(\sigma-\sigma_{u}\right)-m \ln \sigma_{0}
$$
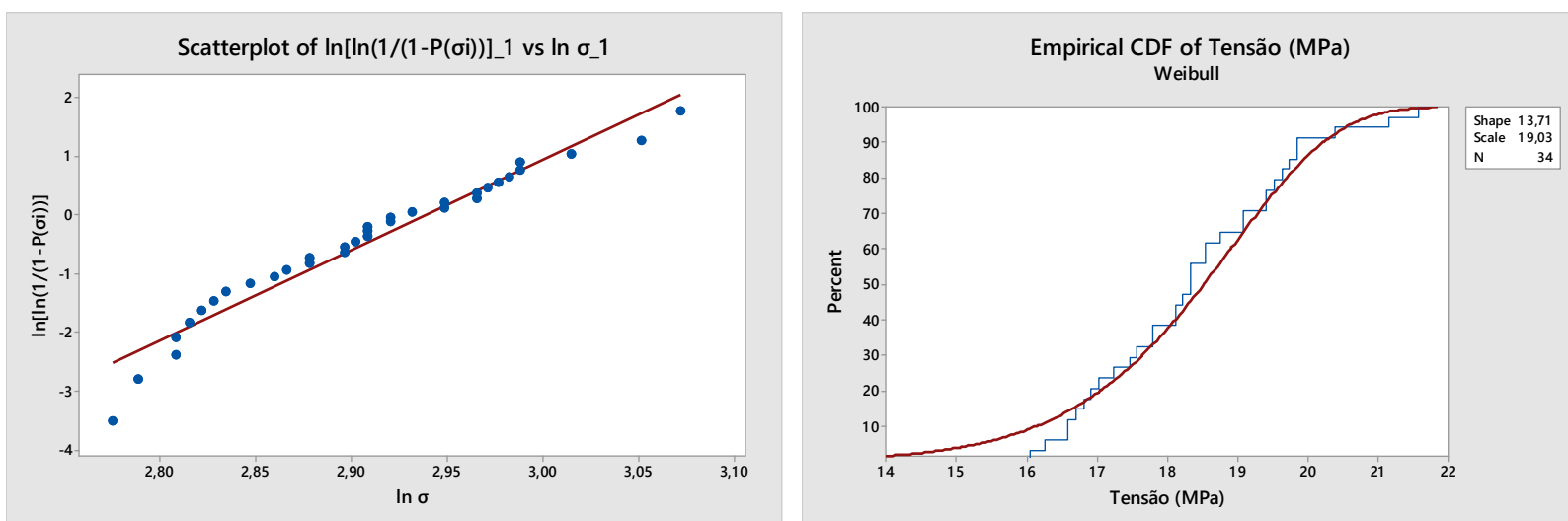

Figura 7. À esquerda: dispersão dos pontos Weibull $2 p$ com reta ajustada. À direita: função de distribuição acumulada de Weibull $2 p$.

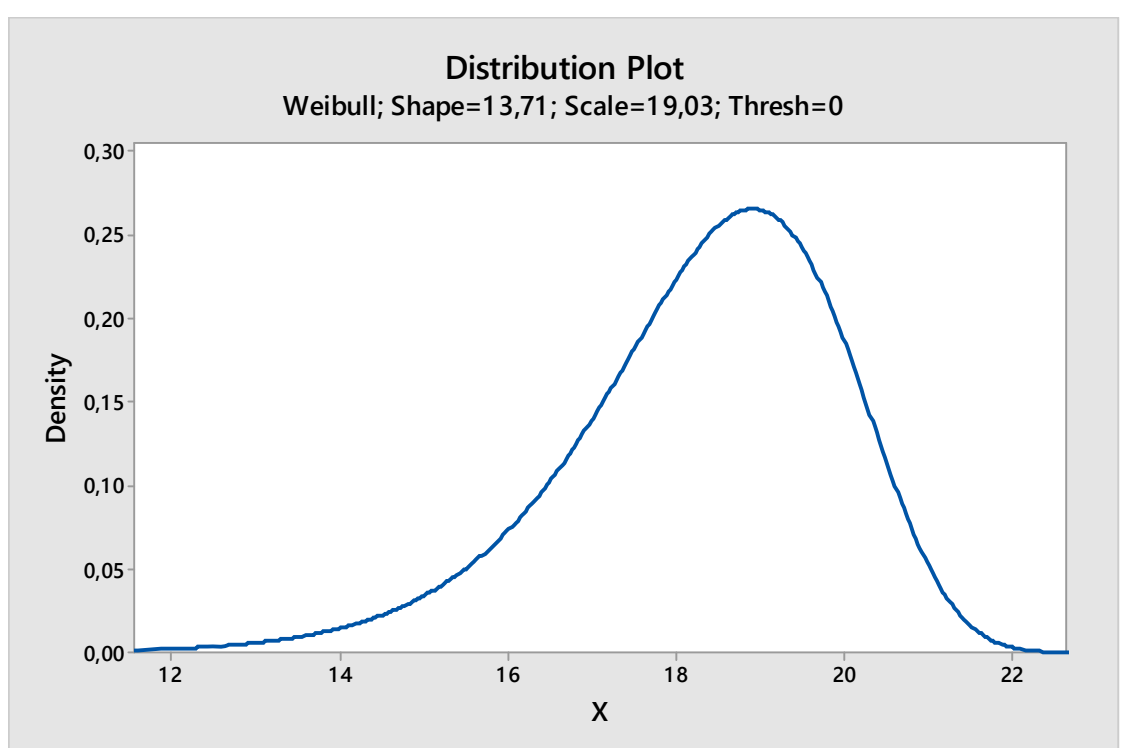

Figura 8. Função distribuição de probabilidades de Weibull $2 p$ função.

Para a determinação dos coeficientes da equação (10), é necessário ainda calcular os valores de $\ln (\sigma \mathrm{i})$ e $\ln [\ln (1 /(1-\mathrm{Pi})]$ apresentados na Tabela 8. Mantendo os mesmos procedimentos adotados na regressão utilizada para determinar os dois parâmetros da distribuição de Weibull, foi obtida a reta ajustada conforme gráfico da Figura 9. Através deste ajuste foram determinados os valores dos três parâmetros de Weibull, módulo $m=2,02$ e o fator de escala $x_{0}=2,98$ e valor de posição, $x_{u}=15,74$. Estes parâmetros foram usados na construção do gráfico de distribuição acumulada de Weibull, também mostrado na Figura 9 e o gráfico de distribuição de probabilidades mostrado na Figura 10. 
Tabela 8. Dados calculados para $2^{\mathrm{a}}$ Regressão Linear do $2^{\circ}$ ensaio.

\begin{tabular}{cccccc}
\hline $\mathbf{C P}$ & $\ln \left(\boldsymbol{\sigma}-\boldsymbol{\sigma}_{\mathrm{u}}\right)$ & $\operatorname{In}[\operatorname{In}(\mathbf{1} /(\mathbf{1}-\mathrm{Pi})]$ & $\mathbf{C P}$ & $\ln \left(\boldsymbol{\sigma}-\boldsymbol{\sigma}_{\mathrm{u}}\right)$ & $\operatorname{In}[\ln (\mathbf{1} /(\mathbf{1}-\mathrm{Pi})]$ \\
\hline 19 & $-1,14649$ & $-3,51147$ & 8 & 0,95384 & $-0,28267$ \\
33 & $-0,62614$ & $-2,80305$ & 22 & 0,95384 & $-0,20051$ \\
4 & $-0,15064$ & $-2,38192$ & 16 & 1,03421 & $-0,11957$ \\
$\ldots$ & $\ldots$ & $\ldots$ & $\ldots$ & $\ldots$ & $\ldots$ \\
26 & 0,86658 & $-0,54142$ & 5 & 1,53837 & 1,04141 \\
18 & 0,91123 & $-0,45257$ & 31 & 1,68941 & 1,26027 \\
7 & 0,95384 & $-0,36651$ & 23 & 1,76646 & 1,76284
\end{tabular}
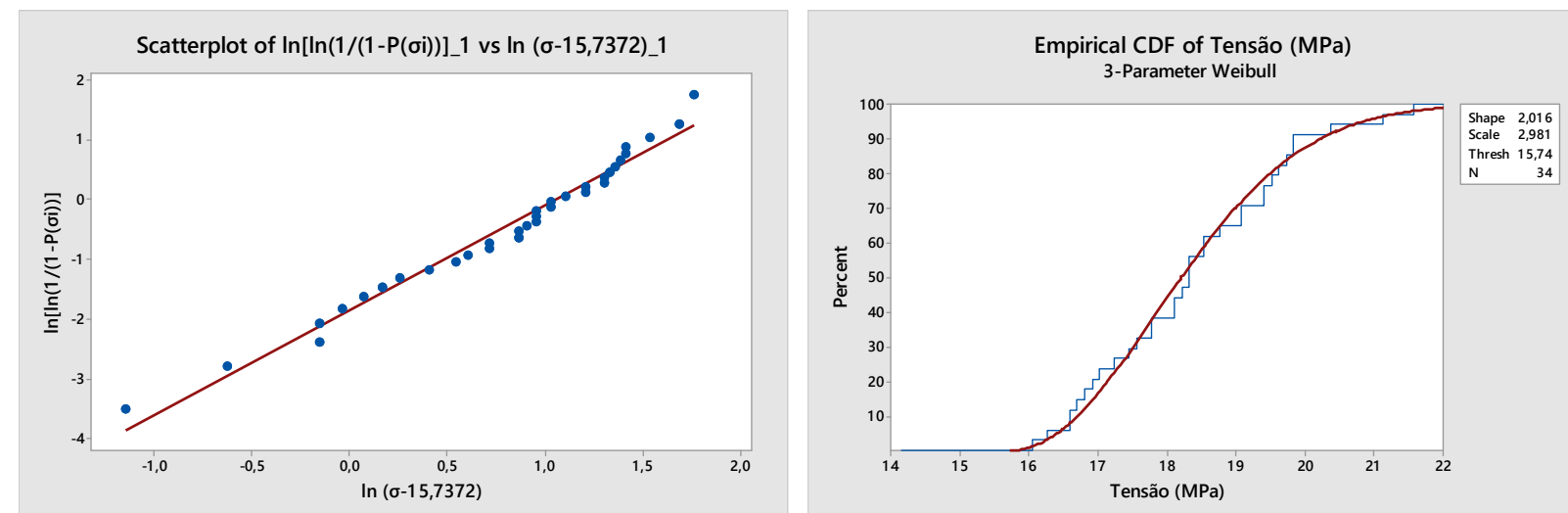

Figura 9. À esquerda: Dispersão dos pontos Weibull 3p com reta ajustada. À direita: Função de distribuição acumulada de Weibull 3p.

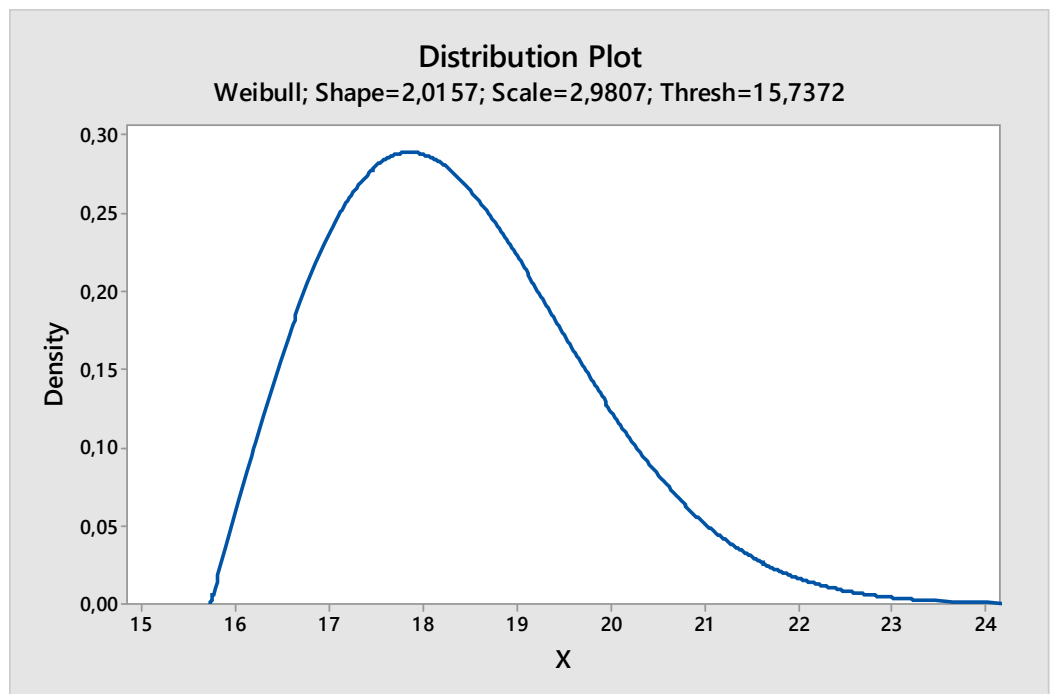

Figura 10. Função distribuição de probabilidades de Weibull 3p

\subsection{Análise dos Resultados da fdp de Weibull com 2 e 3 Parâmetros}

Observando as regressões dos dados da distribuição para Weibull com dois e três parâmetros percebe-se um melhor ajuste com o uso de três parâmetros, que passou a ser adotada nas análises. Para analisar esses resultados, o principal valor a ser considerado é o coeficiente de Weibull $(\mathrm{m})$. A mais importante conclusão obtida com o valor de m é que, quanto maior o seu valor, maior é acurácia, ou seja, a dispersão tende a ser menor, facilitando para que a curva da Distribuição de Weibull tenha a forma de pico. Como resultado, se obtém uma distribuição mais homogênea dos 
defeitos na microestrutura do corpo de prova. Os resultados de resistência mecânica demonstraram que os pisos cerâmicos apresentaram um bom valor de módulo de resistência à flexão, o módulo de Weibull obtido, $m=2,02$, para todos os CPs foi significativo, o que demonstra a reprodutibilidade da resistência mecânica das cerâmicas.

\section{CONCLUSÃO}

Após as análises e estudos concluímos que todos os corpos de provas tiveram um comportamento bastante similar, ou seja, a composição de sua matéria prima principal não permitiu grandes variações; a absorção de água em um corpo cerâmico está relacionada à sua porosidade, quanto menor for o valor desta maior será sua resistência, e as três amostras submersas em água, não demonstram uma tendência de maior flexão, indicando a necessidade de um tempo maior em submersão na água para a verificação do possível novo comportamento, o que sugere a realização de novos trabalhos.

Os ensaios para os CP's 04 até o 37, com velocidade a taxa de $0,21 \mathrm{MPa} / \mathrm{s}$ apresentaram dois feixes de linhas com inclinação bastante semelhante o que sugere um comportamento similar, seria muito interessante a realização de um estudo futuro para averiguação das características de fratura dessas cerâmicas.

\section{REFERÊNCIAS}

1 ANAFACER (Associação Nacional dos Fabricantes de Cerâmica para Revestimentos, Loucas Sanitárias e Congêneres). O Mercado Brasileiro. Disponível em: http://www.anfacer.org.br/site. Acesso em 22 de junho de 2014.

2 ABC - Associação Brasileira de Cerâmica. Informações Técnicas - Definição e Classificação. Disponível em: http://www.abceram.org.br/site/?area=4. Acesso em 22 de junho de 2014.

3 Barnett, V.; Lewis, T. Outliers in statistical data. Chichester: John Wiley, 1995. 584 p.

4 Hoaglin, D. C.; Mosteller, F.; Tukey, J.W. Análise exploratória de dados: técnicas robustas - um guia. Salamandra: Almada, 1992. 446 p.

5 INSPEBRAS. Disponível em: http://www.inspebras.com.br/index.php. Acesso em 17 de junho de 2014.

6 NBR 13818 - Placas cerâmicas para revestimento - Especificação e métodos de ensaios. 1997. ABNT - Associação Brasileira de Normas Técnicas.

7 National Institute of Standards and Tecnology-NIST. Statistics for Scientists \& Engineers: exploratory data analysis. Disponível em: http://www.itl.nist.gov. Acesso em 20 de agosto de 2014.

8 Imasava, F. J.; Gouvêa, M. T.; Xavier, C.; Rodrigues, S. Uso do microcomputador nos cálculos do método estatístico de Weibull para a medida da resistência dos materiais frágeis. Anais do $33^{\circ}$ Congresso Brasileiro de Cerâmica da Associação Brasileira de Cerâmica. p. 985-1000, 1989.

9 Wang, Y. et al. Application of Weibull distribution analysis to the dieletric failure of multilayer ceramic capacitors. Materials Science \& Engineering B. n. 47, p. 197-203, 1997.

10 W.J.Dixon(1953). “Processing Data for Outiliers”, Biometrics, 9(1) 74-89. Disponível em: http://webspace.ship.edu/pgmarr/Geo441/Readings/Dixon\%201953\%20\%20Processing\%20Data\%20for\%20Outliers.pdf. Acesso em 20 de agosto de 2014. 